\title{
Hippocampal Dynamics Predict Interindividual Cognitive Differences in Rats
}

\author{
Vincent Hok, ${ }^{1}$ Ehsan Chah, ${ }^{1,2}$ Richard B. Reilly, ${ }^{1,2}$ and Shane M. O’Mara ${ }^{1}$ \\ ${ }^{1}$ Trinity College Institute of Neuroscience and ${ }^{2}$ Trinity Centre for Bioengineering, Trinity College Dublin, Dublin 2, Ireland
}

There are important and sustained interindividual differences in cognition during aging. Here, we investigated hippocampal spatial representations in a rat model of cognitive aging characterized by individual differences in a mnemonic task. Individual cognitive capabilities in old rats were assessed in a delayed non-matching-to-position task. We recorded hippocampal CA1 place cells as the rats explored a familiar environment. Unlike the usual place cells commonly described in the literature, we found that a significant fraction of pyramidal neurons recorded in our study showed a substantial delayed onset of their place field activity. We established that this firing onset delay naturally occurs under basal conditions in old rats and is positively correlated with the remapping status of the animals. The lack of firing during the first few hundred seconds after the animals were introduced into a familiar environment was also associated with an increased locomotion in the remapping rats. This delayed activity is central to understanding the individual basis of age-related cognitive impairment and to resolving numerous discrepancies in the literature on the place cell contribution to the etiology of agedrelated decline. Finally, we also found a positive correlation between the degree of firing variability of place cells ("overdispersion") and performance during the long delays in the delayed non-matching-to-position task. Place cell overdispersion might provide the functional basis for interindividual differences in behavior and cognition.

\section{Introduction}

Place cells are pyramidal neurons in the hippocampus selectively activated while the animal's head is in a particular location in a particular environment (O’Keefe and Dostrovsky, 1971). Place cells are therefore characterized by location-specific firing ("place fields"), and their activity is subject to, among other variables, environmental manipulations (O'Keefe and Conway, 1978; Muller and Kubie, 1987). In young rats, environments are reliably encoded by groups of neurons displaying place fields that can be stable for up to several months (Thompson and Best, 1990). Contrasting with this strong spatial reliability, Fenton and Muller (1998) demonstrated the existence of a striking discharge variability from one pass to another as the rat goes through a firing field. This variability, termed "overdispersion," correlates with the cognitive load of the behavioral task (Jackson and Redish, 2007; Johnson et al., 2009; Fenton et al., 2010) and may constitute a neurophysiological marker for individual cognitive ability (Rapp and Amaral, 1992). We therefore examined overdispersion characteristics of CA1 place cells in old rats and their individual performance in a delayed non-matching-to-position (DNMTP) task. Old rats were chosen because this age category is more likely to display important interindividual cognitive perfor-

\footnotetext{
Received Dec. 24, 2011; accepted Jan. 13, 2012.

Author contributions: V.H. designed research; V.H. performed research; V.H. and E.C. analyzed data; V.H., E.C., R.B.R., and S.M.O'M. wrote the paper.

The authors declare no competing financial interests.

Correspondence should be addressed to Vincent Hok, Trinity College Institute of Neuroscience, Trinity College, Dublin 2, Ireland. E-mail: hokv@tcd.ie.

DOI:10.1523/JNEUROSCI.6449-11.2012

Copyright $\odot 2012$ the authors $\quad 0270-6474 / 12 / 323540-12 \$ 15.00 / 0$
}

mance variability compared with young rats (Ingram, 1988; Gallagher et al., 1993).

In general, no major differences have been found between the basic properties of place cells (average and peak firing rates, interspike intervals, spike amplitude, and width) in young and old animals (Barnes et al., 1983; Markus et al., 1994; Mizumori et al., 1996; Mizumori and Kalyani, 1997). Clear-cut differences between young and old rats, however, do arise when evaluating place field dynamics (Barnes et al., 1997; Shen et al., 1997; Wilson et al., 2003). For instance, Barnes et al. (1997) reported that place field representations in young rats were reliable both within and between episodes in a familiar environment, whereas in old rats, spatial representations were stable during an episode but frequently exhibited complete rearrangements ("global remapping") between episodes. Contrasting with this apparent withinsession reliability, we find in old rats a substantial proportion of hippocampal place cells showing a late development of firing once the animals were introduced into a familiar environment. More importantly, this late-onset activity usually occurred in animals who completely remapped between sessions in the same environment. This phenomenon is similar to the one either identified but noted tangentially (Hill, 1978; Tanila et al., 1997a,b) or tacitly investigated (Wilson and McNaughton, 1993; Leutgeb et al., 2004) when young rats explore a novel environment. When tested in a DNMTP task, the remapping rats were impaired specifically in the long delays sensitive to hippocampal damage (Hampson et al., 1999) and aging (Dunnett et al., 1988). Finally, the overdispersion analysis revealed a positive correlation between the degree of variability in place cell firing and individual performance in the memory task at longer delays, thereby providing a new way to investigate cognitive function in individuals. 


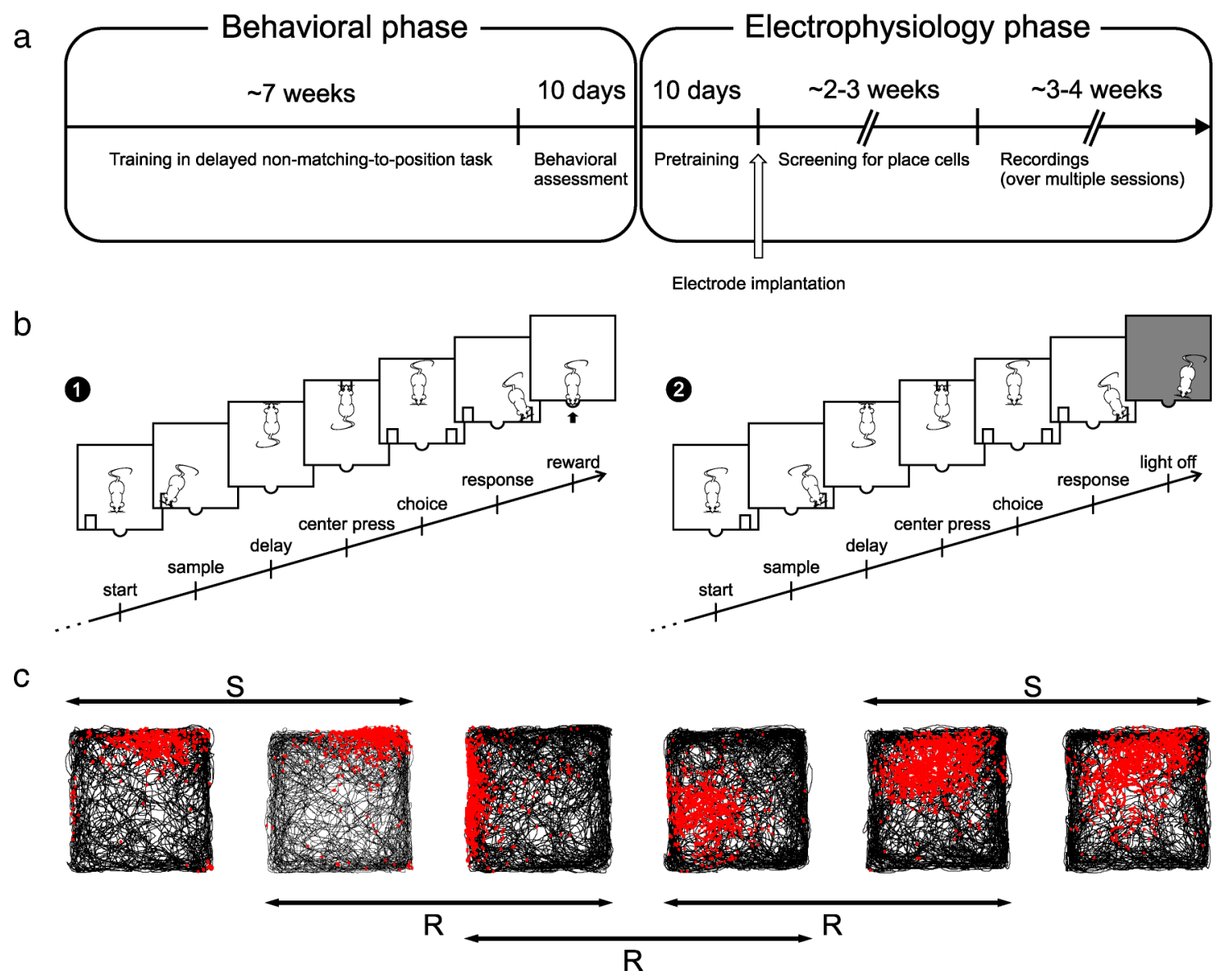

Figure 1. Schematic summarizing the details of the procedure. $\boldsymbol{a}$, Schematic showing the different phases of the procedure over the course of the experiment. $\boldsymbol{b}$, Experimental design of the DNMTP task. The sequence depicted on the left ((-) represents a correct trial rewarded by a sucrose pellet (arrow), and the sequence on the right (2) represents an error trial ended without reward delivery and the house light switched off. The sample phase in which a single lever (left or right) is extended and pressed (sample) is followed immediately by a delay phase (1-30 s duration) initiated by sample lever press and requiring a lever press on the opposite wall at the end of the delay (center press). The non-match phase is initiated by center press after the delay times out. Both levers are presented, and the non-match response (response) on the lever opposite the sample is reinforced with a sucrose pellet. A response on same lever as the sample is an error. $c$, Illustrative example of one neuron recorded over multiple sessions (separated at $\geq 4 \mathrm{~h}$ ). The maps represent for each session the trajectory of the rat (black line) with superimposed spike locations (red). $\mathrm{S}$ denotes a stable event (place field activity is at the same location from one session to the other), and R indicates a remapping event (the location of the place field is uncorrelated with the previous session). In this example, five pairs of sessions are identified: two stable and three unstable (thus giving a remapping proportion of $60 \%$ ).

\section{Materials and Methods}

Subjects

Subjects were male Han Wistar rats ranging in age from 14 to 18 months $(n=5)$ and from 24 to 30 months $(n=5)$ at the time of testing. All experimental procedures (behavioral, electrophysiological, and surgical) adhered to the relevant European Union (86/609/EEC) and national guidelines.

\section{Operant Task Sessions}

Apparatus. Experiments were performed in a Plexiglas behavioral testing chamber $(30 \times 25 \times 25 \mathrm{~cm}$ high; Med Associates) with two retractable levers mounted on one wall $5 \mathrm{~cm}$ above the chamber floor (distance between levers is $12 \mathrm{~cm}$ ), a hopper $(5 \times 5 \mathrm{~cm})$ mounted between the levers where $45 \mathrm{mg}$ dustless sucrose pellets are delivered (5TUT formula; TestDiet), and a retractable lever mounted on the center of the opposite wall $5 \mathrm{~cm}$ above the chamber floor.

Behavioral paradigm. The DNMTP task requires the animal to press the retractable lever presented on a random basis in the left or right position (sample response) to initiate the trial (Fig. 1). This initiates a delay phase randomly timed between 1 and $30 \mathrm{~s}$, at the end of which the animals have to press the center lever located on the opposite wall. Animals then return to where now both left and right levers are extended (match/non-match phase). The correct response requires a press on the opposite lever pressed during the sample phase, constituting the nonmatch response, which is followed by delivery of a sucrose pellet into the hopper. An incorrect response (pressing the same lever as the one pressed in the sample phase) produces a $5 \mathrm{~s}$ timeout in which the overhead light is turned off. Trials are separated by $10 \mathrm{~s}$. Overall performance of the task declines in a linear manner as the duration of the delay interval is increased from 1 to $30 \mathrm{~s}$. This task is sensitive at long delays to hippocampal disruption (Hampson et al., 1999) and aging (Dunnett et al., 1988).

Freely moving sessions

Once the DNMTP task had been performed for $60 \mathrm{~d}$, the animals were trained in a pellet-chasing task for $10 \mathrm{~d}$ (see below) and then implanted with a bundle of eight tetrodes. The recordings took place in a square arena $(64 \times 64 \times 25 \mathrm{~cm}$ high) located in the center of a room with several multimodal cues available (visual, olfactory, and auditory). Rats were food deprived to $80 \%$ of their original weight. Subjects were then placed in the open field and $20 \mathrm{mg}$ food pellets (5TUL formula; TestDiet) were thrown in every $20 \mathrm{~s}$ to random locations within the open field; in this way, animals were in continuous locomotion, allowing for complete sampling of the environment. Each recording session lasted $20 \mathrm{~min}$, and consecutive sessions were at least $4 \mathrm{~h}$ apart. Rats were returned to their home cage between two sessions (for a summary, see Fig. 1).

Surgical implantation of electrodes. Recording electrodes were eight bundles of four platinum-iridium wires (90\% platinum, 10\% iridium; heavy polyimide enamel insulated, $25 \mu \mathrm{m}$ bare wire diameter; California Fine Wire) twisted together. Tetrodes were threaded through a guide cannula, 25 gauge, and protected with 21 gauge cover. Tetrodes were 
then mounted on a lightweight microdrive (Axona), cut flat, and implanted in the dorsal hippocampus $(-3.2 \mathrm{~mm}$ anteroposterior, \pm 3.0 $\mathrm{mm}$ mediolateral, and $1.5 \mathrm{~mm}$ dorsoventral to dura). The microdrives are built around a precision screw, machined to a pitch of $200 \mu \mathrm{m}$, which advances the electrodes in $25 \mu \mathrm{m}$ steps (one-eighth turns). A spring tensions the screw and prevents it from moving spontaneously, therefore allowing recording of the same cell over days.

Recording techniques. After a minimum 1 week recovery, subjects were connected, via a 32 channel head stage (Axona), to a recording system that also allowed for animal position tracking. Signals were amplified between 3000 and 12,000 and bandpass filtered between $380 \mathrm{~Hz}$ and 7 $\mathrm{kHz}$ for single-unit detection. To maximize unit separation, only waveforms of sufficient amplitude (at least three times noise threshold) were acquired. Candidate waveforms were then discriminated offline using graphical cluster-cutting software (TINT; Axona), which allows waveform separation based on multiple features. Such features include spike amplitude, spike duration, maximum and minimum spike voltage, and the time of occurrence of maximum and minimum spike voltages. It was also possible for the experimenter to discriminate the waveforms according to the voltage at a specified time. Using custom-written programs (MATLAB), autocorrelation histograms were built for each unit, and the whole unit was removed from analysis if the histogram revealed the existence of correlations within the first $2 \mathrm{~ms}$ (refractory period), inconsistent with good unit isolation. Similar to other studies (Leutgeb et al., 2007; Fenton et al., 2010), place cells were selected for study if their spatial firing patterns were location specific (coherence $\geq 0.25$; spatial information $\geq 0.5$ bits/action potential) and robust (average activity $\geq 0.25 \mathrm{~Hz}$ ).

Spatial firing analysis. Firing rate maps allow for visual inspection of the preferred areas of firing for the neurons (i.e., place fields). They were constructed by dividing the number of spikes that occurred in specific pixel coordinates by the total trial time the animal spent in that coordinate. This produced maps depicting the place fields of each cell in Hertz. The pixel map is converted into a $22 \times 22$ array of square bins $3 \mathrm{~cm}$ on a side. Autoscaled color-coded firing rate maps were then created to visualize firing rate distributions (Muller et al., 1987). In such maps, pixels in which no spikes occurred during the whole session are displayed as blue. The highest firing rate is coded as red, and intermediate rates are shown as orange, yellow, green, and cyan pixels from high to low. We used multiple indices to analyze the spatial properties of the hippocampus place cell firing (namely spatial coherence, spatial information content, and spatial reliability). A firing field was defined as a set of at least nine contiguous pixels with firing rate above zero. Only the largest field is considered for each cell if more than one field was found. The center of the field was defined as the $3 \times 3$ group of pixels with the greatest mean rate (Fenton et al., 2000). Spatial coherence consists of a spatial autocorrelation of the place field map and measures the extent to which the firing rate in a particular bin is predicted by the average rate of the eight surrounding bins. Thus, high positive values resulted if the rate for each bin could be better predicted given the firing frequency of the neighbor location (Muller and Kubie, 1989). The spatial information content is expressed in bits per spike (Skaggs et al., 1993) and is calculated as follows:

$$
I=\sum_{i} P_{i}\left(\lambda_{i} / \lambda\right) \log _{2}\left(\lambda_{\mathrm{i}} / \lambda\right)
$$

where $\lambda_{\mathrm{i}}$ is the mean firing rate in bin $i, \lambda$ is the overall mean firing rate, and $P_{\mathrm{i}}$ is the occupancy probability of bin $i$. In short, the spatial information content index can be seen as a measure of the amount of information relative to the location of the animal conveyed by a single action potential emitted by a single place cell. Finally, spatial reliability was calculated by measuring the cross-correlation (Pearson's coefficient correlation) between the smoothed (using a $5 \times 5$ kernel) firing rate maps of a cell for the first $10 \mathrm{~min}$ and the last $10 \mathrm{~min}$ of a recording session.

To assess the remapping status of individuals, we performed crosscorrelations between smoothed firing rate session maps in which the same neurons are identified based on their waveform properties (see below). To be considered as a remapping event, the correlation had to fall under a cutoff of $r=0.5$ (Barnes et al., 1997; see also Fig. 5b); the animal was considered a remapping rat only if those events represented $>5 \%$ of the overall correlations. To identify the sameness of the neurons, the waveforms recorded on the same electrode across different sessions were compared, based on the method used by Grossman et al. (2008). Briefly, multivariate ANOVAs using the Wilks' $\lambda$ statistic allowed the comparison of the waveform recorded in the same tetrode between two consecutive sessions. As a control comparison, 365 non-delayed place cells with a strong spatial reliability index (Pearson's coefficient correlation within session $>0.9$ ) were used to construct a probability distribution of the $\lambda$ values obtained by comparing the waveforms between the first 10 and the last $10 \mathrm{~min}$ of the session. Waveforms were only described as coming from the same single neuron across sessions if the Wilks' $\lambda$ value comparing the two sessions was in the same range as those for the withinsession comparisons (Fig. 2b,c).

Characterizing delayed activity. To be identified as a late-onset activity neuron, the place cell had to be silent during at least the two first passes through the center of the place field (see below, Quantifying overdispersion). The time stamp related to the onset of the activity is extracted from the first active pass that is immediately followed by another non-zero firing pass. In this way, classifying one cell into the non-delayed category does not bias the main measure characterizing the onset activity. For example, one cell that has been identified as non-delayed (i.e., a cell that is active during at least one of the first two passes) can show an onset greater than the one related to the first pass (e.g., two consecutive active passes occur only at the eighth pass). However, considering now the cells identified as delayed, this onset index is biased toward upper values; the time of onset is therefore provided solely for the sake of comparison between subgroups of the same category.

To rule out the possibility of a directional bias as a possible source of delayed place cell activity, we analyzed the place $\times$ direction interaction using the distributive ratio hypothesis developed by Muller et al. (1994). This approach has been preferred to the one developed by Caccucci et al. (2004) because the latter relies on the Poisson distribution assumption to estimate the firing of the cell on the occupation time basis (on this specific matter, see Burgess et al., 2005). Place cell directionality was first described by McNaughton et al. (1983) while rats were performing a forced choice task in a radial-arm maze. It is assumed that the directionality component of the activity of the place cell is mainly observed in constrained environments rather than in open-field arenas similar to the one used in the current study (McNaughton et al., 1983; Muller et al., 1994; Markus et al., 1995). However, it appeared that, when the animals exhibit a certain stereotypy in their trajectories in an open environment, place cells tend to be more directional than when the animals forage randomly (Markus et al., 1995). Although no intent was made to train the rats in such a way, some animals tend to favor the walls during searching and venture more in the center during chasing (for a similar observation, see Jackson and Redish, 2007). Therefore, one can argue that late-onset activity of certain place cells might be attributable to a strong direction of movement modulation, especially if the animal shows stereotypical trajectories during the initial exploration of the environment.

The distributive hypothesis (Muller et al., 1994) states that the directional firing is only modulated by location and that the only influence of directionality arises from a sampling bias. The predicted rate as a function of direction can be described as follows:

$$
R_{\text {Pred }}(\theta)=\Sigma R_{\mathrm{p}} T_{\mathrm{p}}(\theta) / \Sigma T_{\mathrm{p}}(\theta)
$$

where $R_{\mathrm{p}}$ is the firing rate in one pixel, and $T_{\mathrm{p}}(\theta)$ is the time spent facing direction $\theta$ in that pixel. The test consists of measuring the observed directional tuning for the cell, $R_{\mathrm{Obs}}(\theta)$, against that of the one calculated under the assumption that the effect of direction is purely artifactual, $R_{\text {Pred }}(\theta)$. This is ascertained with the help of a "distributive ratio" (DR):

$$
D R=\Sigma\left|\ln \left(1+R_{\mathrm{Obs}}(\theta)\right) /\left(1+R_{\text {Pred }}(\theta)\right)\right| / N,
$$

where $N$ is the number of directional bins considered. A perfect prediction (i.e., $\mathrm{DR}=0$ ) indicates that the null hypothesis is true, meaning that place cell firing is only modulated by location and the directional influence on place cell firing is only attributable to the inhomogeneity of sampling. Conversely, high values of this ratio would suggest a poor 

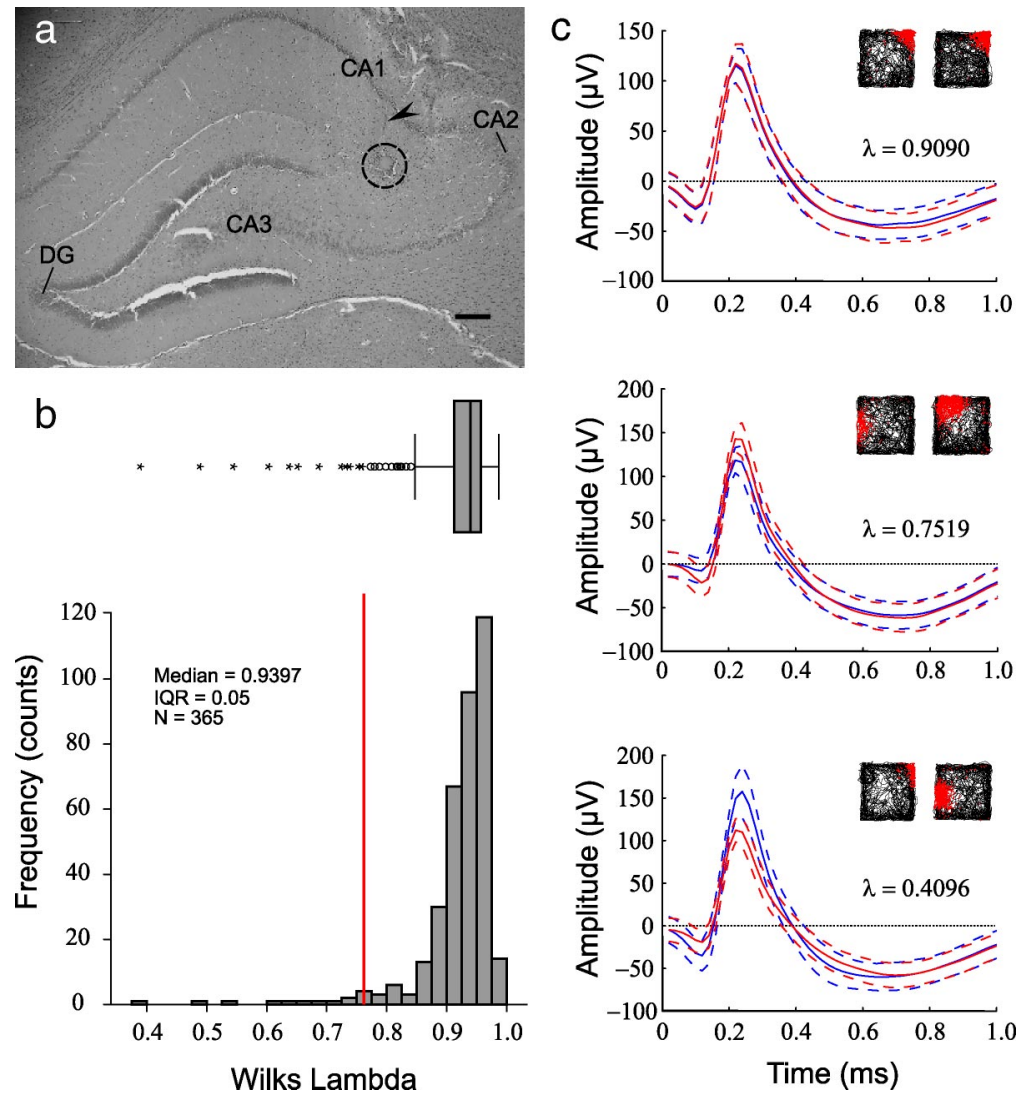

Figure 2. Histological verification and identification of waveforms produced by the same neuron. $\boldsymbol{a}$, Indicative electrode location through CA1. Recording sites were identified by following the electrode track (arrowhead) through serial hematoxylin and eosin-stained sections. The center of the glial reaction indicates the tip of the tetrodes (which were left in place at the end of the experiment; dashed line circle). The exact location of the units recorded during the experiments could therefore be determined using the daily record of electrode position. DG, Dentate gyrus. Scale bar, $250 \mu \mathrm{m}$. $\boldsymbol{b}$, Top, Box plot showing the Wilks' $\lambda$ distribution obtained from 365 pairs of waveforms and pinpointing outliers (circles) and extreme values (asterisks). Outliers are values between 1.5 and 3 times the interquartile range (IQR) from the end of the box, whereas values $>3$ interquartile ranges are defined as extreme (13 of 365). The waveforms were extracted from CA1 hippocampal place cells showing a high reliability measure (Pearson's correlation coefficient within session $>0.9$ ) to ensure good signal stability (for details, see Materials and Methods). Bottom, Frequency histogram based on the Wilks' $\lambda$ distribution. The red line indicates the threshold (0.7623) below which waveforms are considered significantly different from each other and therefore produced by two different neurons. Note that this method does not prevent replicate recordings of the same cell (although when identified, the second cell of the pair has been systematically removed from the main analysis). For this reason, we refer to "spike trains" or "units" rather than "cells" when comparing populations together. $c$, Examples of one matching (top) and two non-matching units (bottom) obtained from rats with stable hippocampal representations. The $\lambda$ value is displayed on the right of each pair of waveforms. For each waveform, the solid line is the average waveform shape, and the dashed lines show the 1 SD confidence intervals. The $y$-axis scale denotes the amplitude of the action potential in microvolts (negativity is up), and the dotted horizontal line through 0 denotes the baseline potential. The length of the $x$-axis represents $1 \mathrm{~ms}$. The corresponding trajectory maps are shown in the top right corner for each pair of waveforms.

prediction (indicating that directionality accounts for some of the variability in the firing rate of the cell).

Quantifying overdispersion. Overdispersion was measured as described by Fenton and Muller (1998). Briefly, passes through the firing field were defined as the time series of positions starting when the light-emitting diode (LED) entered the field and ending when the LED left the field. To enhance the reliability of firing rate estimates, passes were studied only if they met the following two conservative criteria: (1) each pass had to last at least $1 \mathrm{~s}$; (2) the pass had to go through the field center. The observed number of spikes fired during a pass was compared with the number of spikes predicted from the session-averaged positional firing rate distribution. The predicted activity during a pass depends only on the specific pixels visited and the time spent in those pixels without regard to the sequence of positions.

For a given pass, the expected number of spikes is given by:

$$
\exp =\Sigma r_{\mathrm{i}} t_{\mathrm{i}}
$$

where $r_{\mathrm{i}}$ is the time-averaged firing rate at position $i$ in the pass through the field, and $t_{\mathrm{i}}$ is the time spent in location $i$ during the pass. According to the Poisson assumption, the SD of the expected numbers of spikes is equal to $\sqrt{\exp }]$. Thus, $Z$ is a standard-normal deviate that measures the SD of the observed discharge (obs) from this expectation for each pass and is calculated as follows:

$$
Z=\frac{(\text { obs }- \text { exp })}{\sqrt{\exp }} .
$$

Therefore, if $Z \geq 1.96$ or $\leq 1.96$, the probability that the observed number of spikes is consistent with the model is $\leq 0.05$. Overdispersion was then measured as the variance of the distribution of $Z$ values computed for a set of passes.

Histology. The rats received a lethal dose of urethane (ethyl carbamate, $1.5 \mathrm{~g} / \mathrm{kg}$, i.p.) and were perfused intracardially with saline, followed by a $4 \%$ formaldehyde solution. The brains were extracted and stored in formaldehyde, and sagittal sections embedded in wax $(20 \mu \mathrm{m})$ were cut and stained with hematoxylin and eosin. Every section was collected for analysis through the relevant part of the hippocampus. The deepest positions of the electrode traces were identified thanks to the glial reaction around the electrodes (Fig. 2a). The electrodes were not moved after the recordings.

\section{Results}

A total of 628 spike trains from place cells showing good location-specific activity (see Materials and Methods) were recorded in eight rats (for details, see Table $1)$. This ensemble showed an average of $1.09 \pm 0.01$ place fields per unit, which is consistent with CA1 place cell responses recorded in an apparatus of such size (Fenton et al., 2008). From this overall population, 140 units were delayed in their onset once the animal was introduced into its environment. Figure $3 a$ shows an example of late-onset activity in a CA1 place cell in which the difference in grand firing rate (average activity within the firing field) between the first and the last $10 \mathrm{~min}$ of the session is nearly threefold. Breaking the session into $150 \mathrm{~s}$ bouts indicates that this particular cell is active only from the third interval onward (Fig. 3b). Overall, the onset of non-delayed units occurs on average between the first and the second pass within the place field ( $1.74 \pm 0.09$ passes), resulting in a latency of $57.31 \pm 3.78 \mathrm{~s}$ to onset of spiking. Regarding the delayed population, the units are active between the sixth and seventh passes $(6.58 \pm 0.36$ passes $)$, giving an average latency of $234.05 \pm 17.74$ $\mathrm{s}$. When analyzing the variability of discharge during individual passes through the firing field of the non-delayed units, we find a variance in the distribution $\left(\sigma^{2}=4.998, \mu=0.04, N_{\text {passes }}=\right.$ $17730)$, similar to those reported previously under analogous experimental conditions (Fenton and Muller, 1998; Jackson and Redish, 2007; Fenton et al., 2010; Fig. 3d, blue curve). The distribution of the delayed unit population, however, is atypical be- 
Table 1. Main characteristics of spatio-selective units per individual

\begin{tabular}{|c|c|c|c|c|c|c|c|c|}
\hline Rat (age) & Status & Units (\# of sessions) & Non-delayed units & Delayed units (\%) & Pairs identified & Remapping pairs (\%) & Number of passes & Overdispersion $\left(\sigma^{2}\right)$ \\
\hline $\mathrm{rh}(\mathrm{ma})$ & $\mathrm{R}$ & $54(21)$ & 41 & $13(24.07)$ & 12 & $2(16.67)$ & 1834 & 4.33 \\
\hline$r p^{a}(\mathrm{ma})$ & $\mathrm{R}$ & $79(31)$ & 56 & $23(29.11)$ & 23 & $9(39.13)$ & 1379 & 4.80 \\
\hline $\mathrm{rd}(\mathrm{a})$ & $\mathrm{R}$ & $201(51)$ & 129 & $72(35.82)$ & 216 & $140(64.82)$ & 4505 & 4.15 \\
\hline ri (ma) & $S$ & $53(26)$ & 48 & $5(9.43)$ & 130 & $0(0)$ & 1591 & 4.61 \\
\hline $\mathrm{rr}^{a}(\mathrm{ma})$ & $S$ & 29 (19) & 26 & $3(10.34)$ & 16 & $0(0)$ & 833 & 5.41 \\
\hline $\operatorname{rj}(\mathrm{a})$ & $S$ & $50(33)$ & 45 & $5(10)$ & 44 & $0(0)$ & 1058 & 8.22 \\
\hline rn (a) & $S$ & $16(9)$ & 15 & $1(6.25)$ & 6 & $0(0)$ & 423 & 8.03 \\
\hline ro (a) & $S$ & $146(48)$ & 128 & 18 (12.33) & 258 & $12(4.65)$ & 6107 & 5.15 \\
\hline $\mathrm{ra}^{b}(\mathrm{ma})$ & U & $11(9)$ & 11 & $0(0)$ & 0 & $N / A$ & 284 & 7.06 \\
\hline$r v^{b}(a)$ & U & $29(10)$ & 27 & $2(6.90)$ & 0 & $\mathrm{~N} / \mathrm{A}$ & 760 & 5.05 \\
\hline
\end{tabular}

ma, Middle-aged; a, aged; R, Remapping; S, Stable; U, Unknown.

${ }^{a}$ Freely-moving data only.

${ }^{b}$ Data included for memory assessment only.

cause it shows a clear bimodality $\left(\sigma^{2}=5.841, \mu=-0.06, N_{\text {passes }}\right.$ $=5248$; Fig. $3 d$, green curve) and a variance significantly greater than the non-delayed unit distribution (Levene's test for equality of variances, $W=32.694, p<0.001)$. This difference is attributable to the overestimate of the actual number of active passes taken into account to calculate the theoretical Poisson distribution for each delayed unit. Contrary to what is expected from normal place cells (Fig. $3 c,(1)$ ), the time series of the standardized firing rates for individual units identified as delayed (Fig. 3c, (2) reveal a marked increase with time, resulting in two clusters of data, one too "low" at the beginning of the session and the other too "high" toward the end. Rescaling the Poisson estimate according to the onset of the cell (taking into account, therefore, the real number of active passes through the field and the actual average activity per bin) produces an important decrease of variance $\left(\sigma^{2}=4.255, \mu=0.00, N_{\text {passes }}=4446\right.$; Fig. $3 d$, orange curve), significantly smaller than the one observed in the raw distribution of the delayed units $(W=75.111, p<0.001)$. Figure 4 shows several place cells recorded simultaneously, some of which were delayed (cell 3) whereas others are not (cells 1, 2, 4, and 5). To further ensure that late-onset activity is not attributable to electrode movement during the recording session or other sampling artifact, we sought to compare the averaged amplitudes of the waveforms of the first 100 spikes emitted for each unit against the averaged amplitude of the 100 last spikes emitted by the same units. A Wilcoxon's signed-rank test for the 140 pairs analyzed for the delayed units was not significant $(232.5 \pm 9.9 \mu \mathrm{V}$ for the first 100 spikes vs $233.7 \pm 9.8 \mu \mathrm{V}$ for the last 100 spikes; $Z=-0.154$, NS). Finally, we analyzed the place $\times$ direction interaction using the distributive ratio hypothesis developed by Muller et al. (1994; see Materials and Methods). The directional distributive ratios appear to be close to zero for the populations of both non-delayed $(0.100 \pm 0.002)$ and delayed $(0.104 \pm 0.004)$ units and are not statistically different $(Z=-1.048$, NS), therefore ruling out the possibility of a directional bias as a possible source of delayed place cell activity.

When comparing individual data, the proportion of delayed units appears to differ greatly from one subject to the other (Table 1). Strikingly, this fraction is strongly positively correlated with the percentage of remapping pairs identified in each rat (Pearson's correlation coefficient, $r^{2}=0.92, p<0.001$; Fig. $5 a$ ). Therefore, subjects showing global remapping from one session to another are more likely to present late-onset place cell activity. This result is further confirmed when comparing the proportion of delayed units comprising remapping pairs to the proportion of non-delayed units (Fig. 5b, inset, (4). Among all session pairs in which we identified the same neurons and with an " $r$ between session" $<0.5$, we examined the number of pairs in which at least one session showed a unit with delayed activity. We found 109 of such pairs from a total of 160 pairs considered, which is well above chance (binomial $Z$ ratio $=4.51, p<0.001$ ). Pairs showing delayed activity among the pairs producing stable correlations are less numerous than expected by chance (26 of a total of 91 ; binomial $Z$ ratio $=-3.98, p<0.001$; Fig. 6). This result indicates that a remapping event is closely associated with the presence of late-onset activity in a fraction of place cells, explaining the strength of the correlation observed in Figure $5 a$. Finally, it is worth mentioning that, once identified, delayed units have a probability at chance of displaying delayed activity in subsequent sessions. Of $>99$ pairs of sessions in which delayed activity was identified in the course of the first session of the pair considered, only 55 units showed such persistence in their late-onset during a second session (a 50\% probability; binomial $Z$ ratio $=1.01$, NS). This result strengthens the idea that delayed place cells do not constitute a particular subpopulation.

Considering now only the non-delayed units, which, because of the absence of artifact in the Poisson estimate, can be used to assess overdispersion in individuals, we find that the variance of the standardized firing rates distribution varies greatly from one subject to another (Fig. $5 c$ ). Notably, the overdispersion indices observed in the remapping rats appear among the lowest values of the distribution. This is confirmed when pooling the standardized firing rates together and comparing the distribution from the stable rats $\left(\sigma^{2}=5.530, \mu=0.04, N_{\text {passes }}=10012\right)$ to the remapping animals $\left(\sigma^{2}=4.310, \mu=0.03, N_{\text {passes }}=7718 ; W=52.694\right.$, $p<0.001$; Fig. $5 e$ ). In comparison, the variances extracted from the middle-aged rats $\left(\sigma^{2}=4.686, \mu=0.02, N_{\text {passes }}=5637\right)$ and from the aged rats $\left(\sigma^{2}=5.144, \mu=0.04, N_{\text {passes }}=12093\right)$ distributions are not significantly different ( $W=1.033$, NS; Fig. $5 d$ ). This last result indicates that age is not the main factor that segregates the two groups of animals, at least on the basis of the firing variability of their place cells. In summary, to analyze properly overdispersion in individual rats, one has to identify putative delayed place cells that increase the variances of the distributions (Fig. 3d).

To further investigate the relationships between onset latency (and onset pass number) and remapping status in rats, we sought to compare the data from remapping and stable rats. Histograms in Figure $7 a$ show a significant difference in the onset latency $(208.78 \pm$ 17.65 vs $319.33 \pm 47.32$ s, respectively; Mann-Whitney $U$ test, $Z=$ 2.203, $p<0.05)$ but no difference in terms of number of passes $(6.50 \pm 0.39$ vs $6.84 \pm 0.88$ passes; $Z=-0.584$, NS) or distance covered before firing onset $(27.38 \pm 2.09$ vs $35.70 \pm 5.94 \mathrm{~m} ; Z=$ 0.924 , NS; data not shown). One possible source of explanation for 

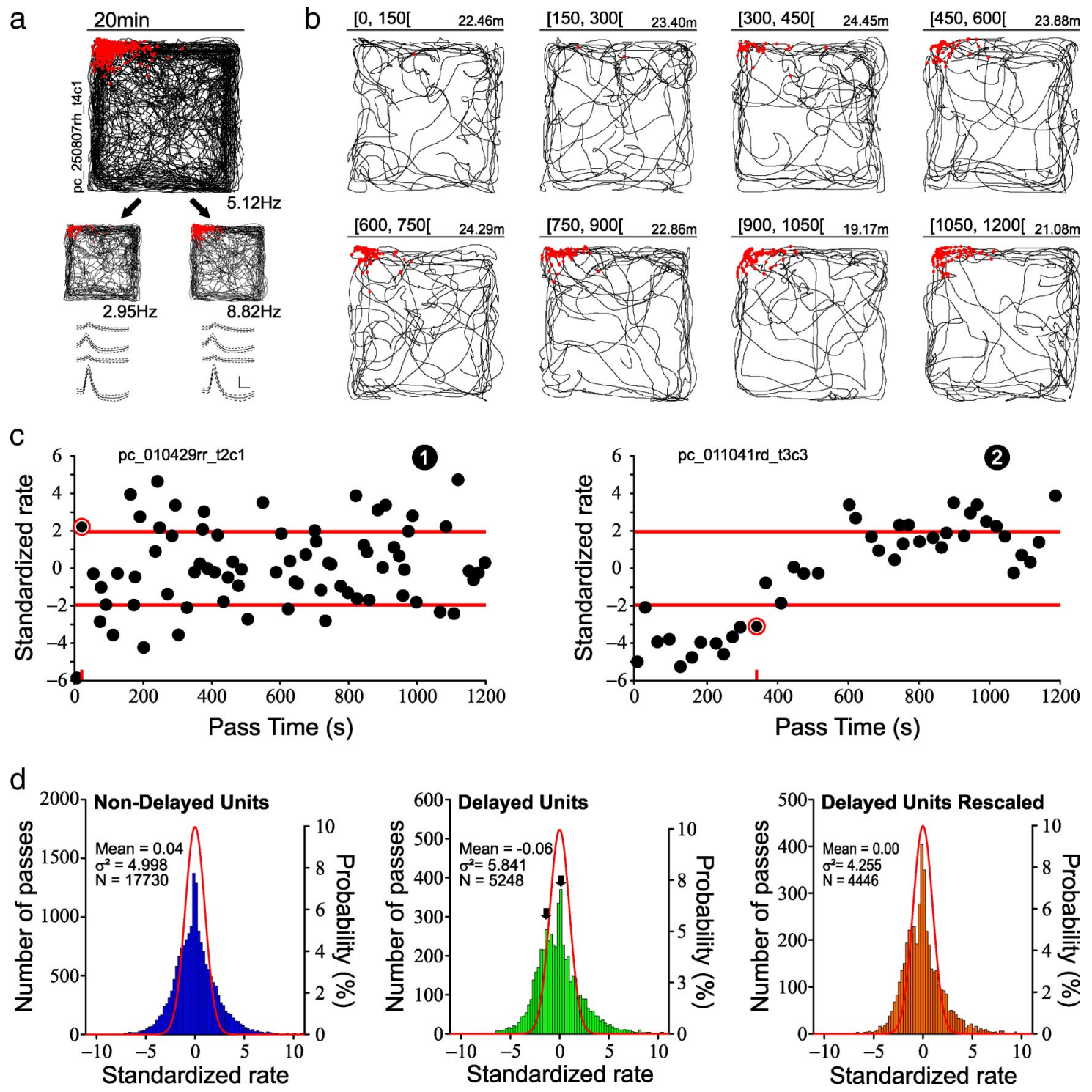

Figure 3. Late place field development in hippocampal CA1 place cells. $\boldsymbol{a}$, Top, Trajectory of the rat (black line) with superimposed spike locations (red), with the grand rate (average activity within the firing field) indicated in the bottom right corner while the animal is foraging in a square arena for $20 \mathrm{~min}$. Bottom, Same cell as previously; the session has been broken down into two halves of $10 \mathrm{~min}$ each. Note the strikingly different firing intensities ( $2.95 \mathrm{~Hz}$ for the first half vs $8.82 \mathrm{~Hz}$ for the second half) despite the place field similarities in location and shape (pixel-by-pixel cross-correlation between the 2 smoothed maps yields a highly significant result: Pearson's correlation coefficient, $r^{2}=0.99, p<0.001$ ). The mean $\pm S D$ spike waveform on each of the four tetrode channels is depicted for the first half (left) and the second half (right) of the session, showing excellent signal stability during the recording sessions. Calibration: $100 \mu \mathrm{V}, 200 \mu$ s. $\boldsymbol{b}$, Late development of CA1 place field in a familiar room (same cell as previously; session has been broken down in 8 blocks of 150 s each). The total distance covered during each time interval appears on the top right corner of the trajectory map. Note the absence of a clearly defined field during the first $300 \mathrm{~s}$ of the session and the subsequent stability of the place field passed this delay. $c$, Plot of the time series of standardized firing rates for the observed firing on all passes through the center of the field of two place cells recorded for $1200 \mathrm{~s}(20 \mathrm{~min}$ ). The red lines indicate the 0.05 probability tails of the normal distribution ( $Z \leq 1.96$ and $Z \geq 1.96)$. Note that, contrary to what is expected from normal place cells ( $\mathbf{(}$; for an example, see Fenton and Muller, 1998), the cell depicted on the right (2) shows increased standardized firing rates over time. For both cells, the standardized firing rate circled in red indicates the first active pass of the cell, and the red tick indicates the time point used to define the latency of firing (for an insight of the development in space and time of this delayed place field, see Fig. 4). $\boldsymbol{d}$, Histograms showing the distribution of standardized firing rates for non-delayed units $\left(N=17,730\right.$ passes through firing fields; left ordinate), delayed units $\left(N_{\text {passes }}=5248\right)$, and delayed units with rescaled activity according to onset latency $\left(N_{\text {passes }}=4446\right)$. The expected distribution is the unit normal distribution drawn in red (right ordinate). For the three distributions, the variance is well above the unitary value expected from a normal distribution, but the distribution from the delayed units is significantly broader than the one based on the non-delayed units (note also the bimodal aspect of the delayed distribution; the 2 peaks are indicated by the black arrows). Rescaling the average activity of the delayed units based on their respective latency significantly reduces the variance when compared with the raw delayed distribution (for details, see Results).

these results lies in the differences in locomotion observed during the session between the two groups. Figure $7 \mathrm{~b}$ shows the amount of distance covered for each group during bouts of $150 \mathrm{~s}$ each. It appears that remapping rats do walk a greater distance than stable animals, at least during the first $300 \mathrm{~s}$ of exploration (segment 1: $20.18 \pm 0.36$ vs $18.60 \pm 0.54 \mathrm{~m}$, respectively; Kruskal-Wallis two-way ANOVA by ranks, $\chi^{2}=5.773, p<$ 0.05 ; segment $2: 20.21 \pm 0.42$ vs $18.12 \pm 0.57 ; \chi^{2}=8.119, p<$ $0.01)$. This last observation is corroborated by a recent exper- iment showing that rats placed in remapping conditions tend to increase their locomotion in a novel environment (Wells et al., 2009). The increased exploration observed in remapping rats co-occurs with the duration of the delay before firing onset. Thus, late onset of place cell firing could mark the end of the remapping process per se.

Finally, we analyzed working memory in eight of the rats before surgery in a DNMTP task (Fig. 8a). Similar to others (Dunnett et al., 1988; Deadwyler and Hampson, 2004), the 
a

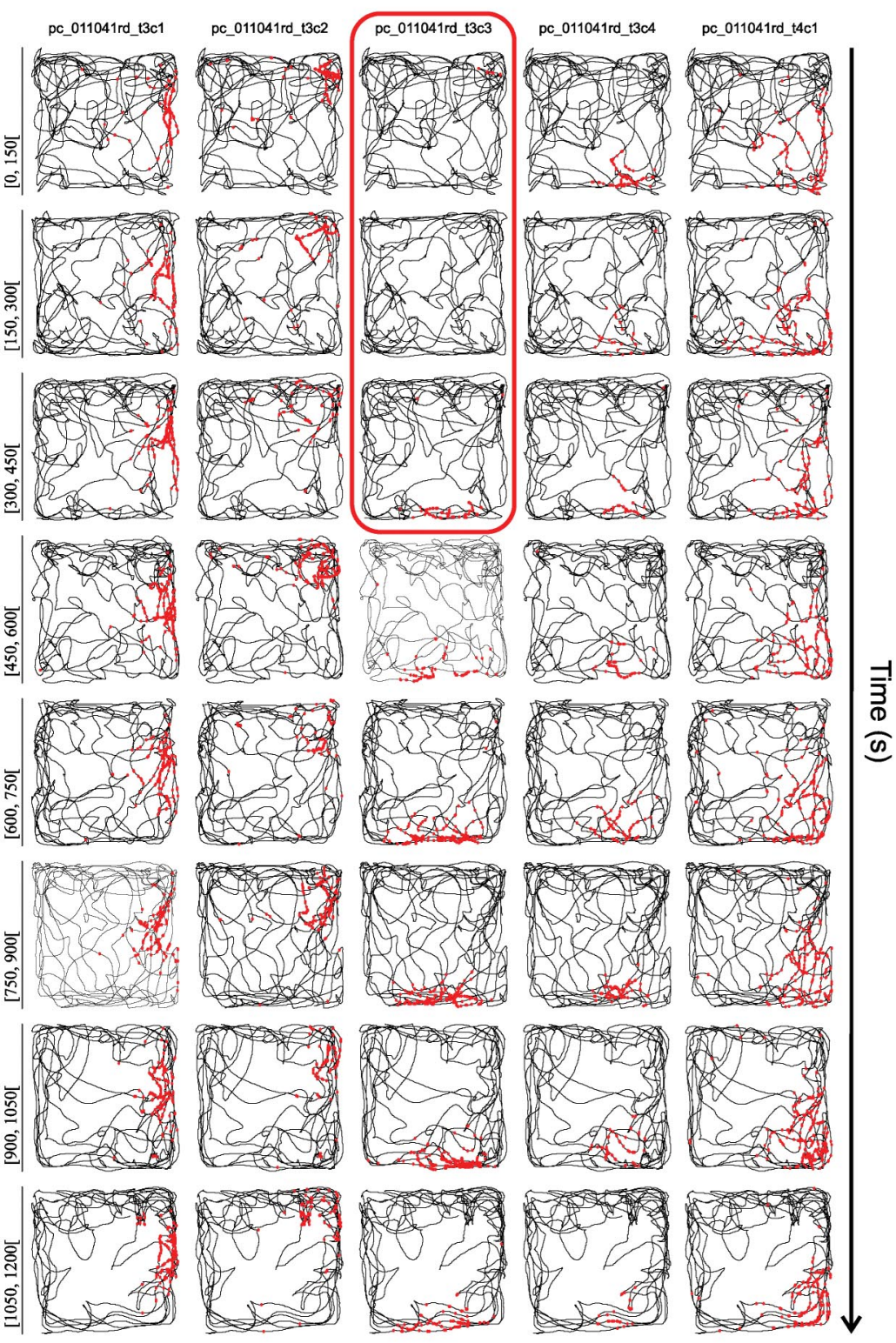

2
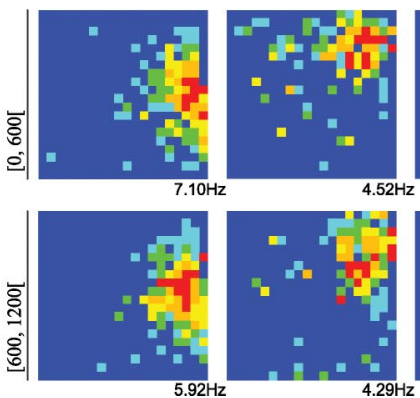

Cell \#2

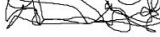

Cell \#3
Cell \#4
Cell \#5

$\overline{3}$
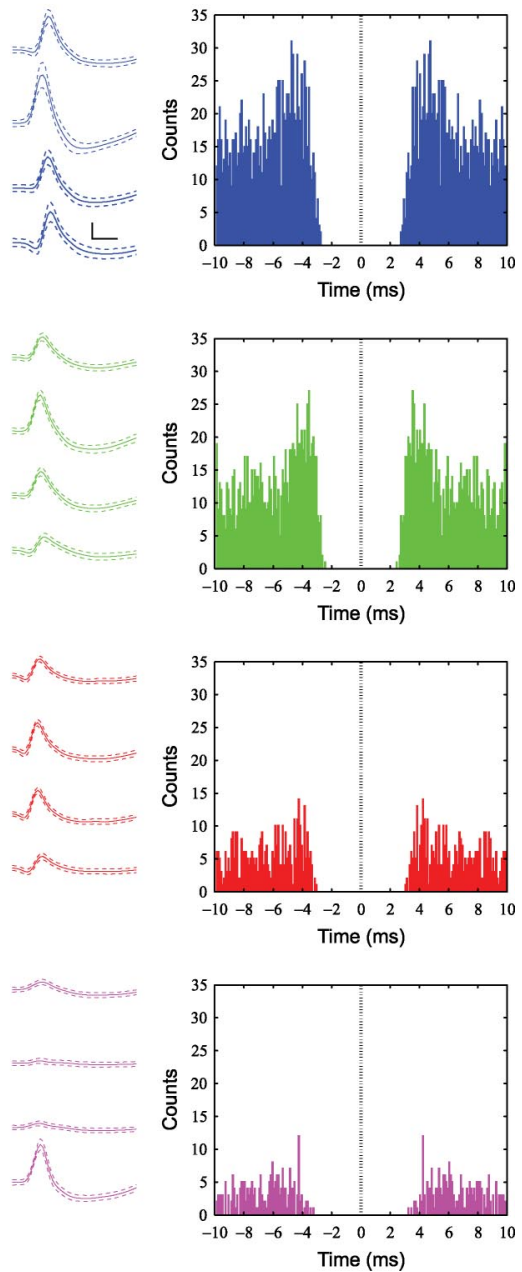

Figure 4. Representative simultaneous recordings of non-delayed and delayed place cells obtained in one rat. $\boldsymbol{a}, \mathbf{0}$, Trajectory maps have been broken down into eight time intervals of $150 \mathrm{~s}$ each. Firing is present from the first bout in Cells $\# 1, \# 2$, \#4, and \#5. Note the delayed-onset of the activity for the third cell (Cell \#3) during the third interval. 2, Color-coded rate maps for the two halves of the session (10 min each) indicating grand rate (average activity within the firing field) in the bottom right corner. Rate map is coded on a color scale from blue (silent) to red (maximum rate), with cyan, green, yellow, and orange pixels as intermediate firing rates from low to high; the number of pixels of a given color is set to 0.8 times the number of pixels in the next lower color. Note the great difference in firing activity for the delayed cell compared with the non-delayed cells. $\boldsymbol{b}$, Multidimensional cluster diagram for the same set of simultaneous recordings as previously. Cluster diagram shows the relationship between peak-to-trough amplitudes of spikes recorded in parallel from electrodes 2 and 3 of one tetrode (t3). Each point represents one sampled signal. Distinct clusters are assigned unique colors. $c$, The mean \pm SD spike waveform on each of the four tetrode channels is depicted on the left with the corresponding autocorrelogram on the right, showing excellent recording isolation (no event recorded within the 2 ms refractory period). Calibration: $100 \mu \mathrm{V}, 200 \mu \mathrm{s}$. 
a

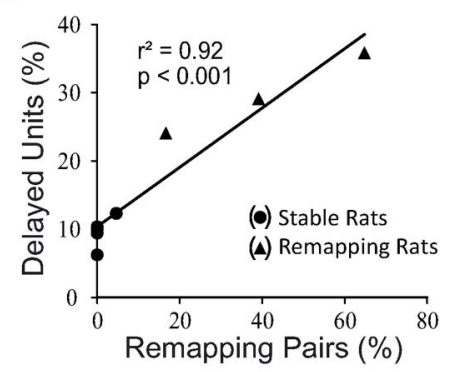

C

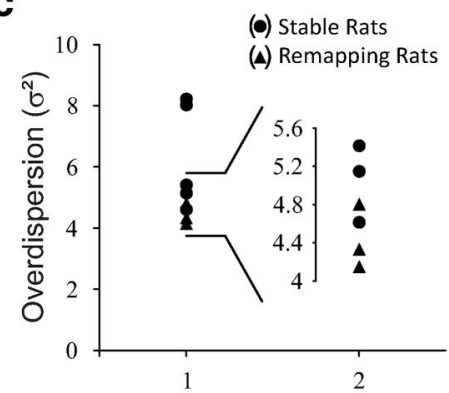

d

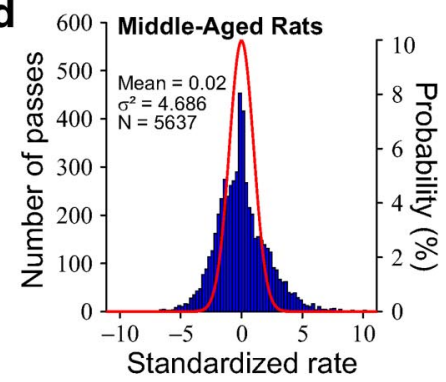

e

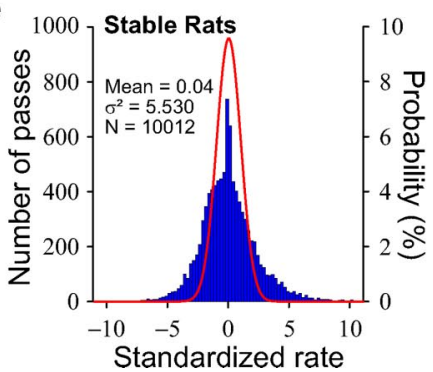

b
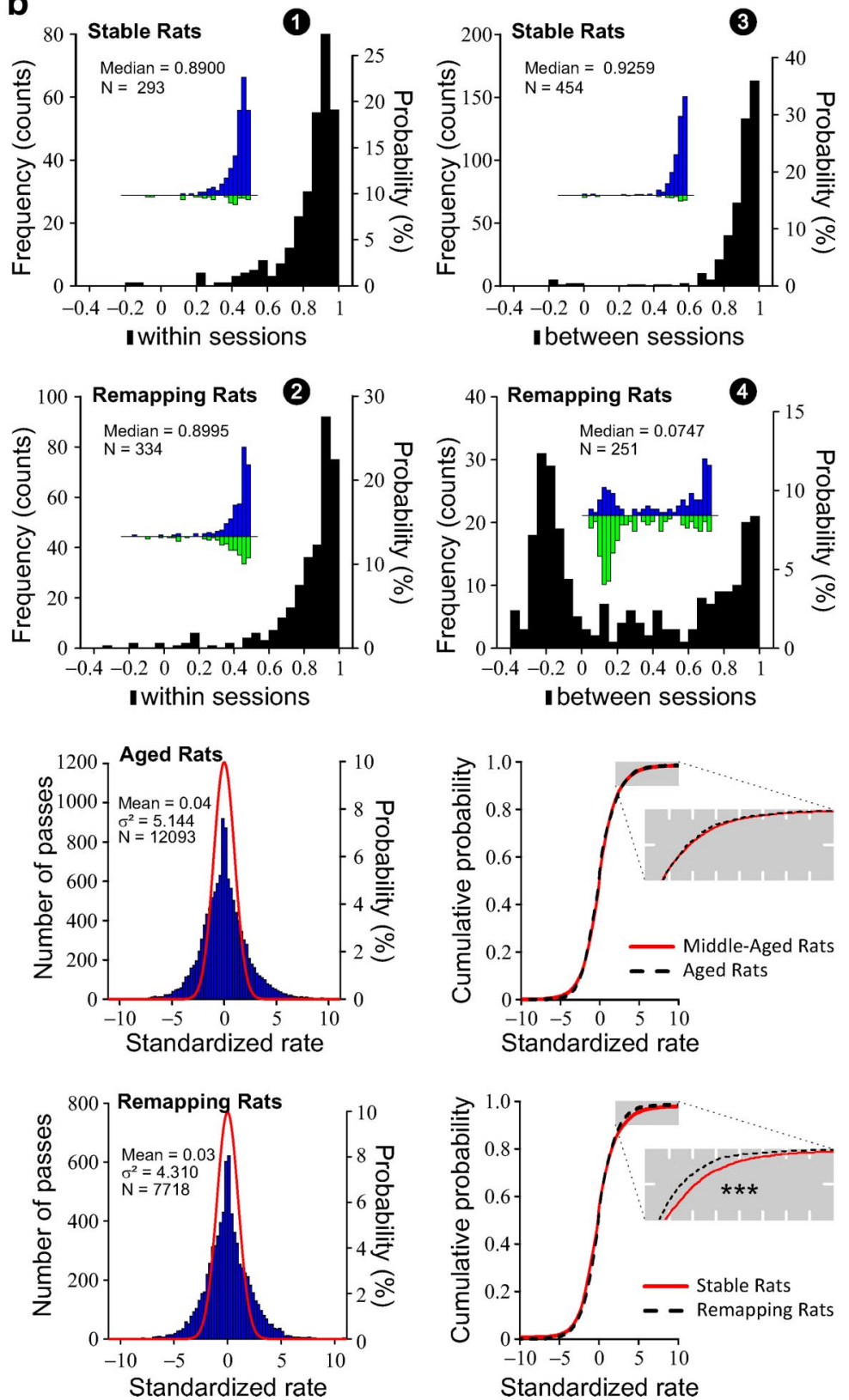

Figure 5. Delayed activity correlates positively with the degree of remapping per individual. $\boldsymbol{a}$, Positive correlation (Pearson's correlation coefficient, $r^{2}=0.92, p<0.001$ ) between the proportion of delayed units and the degree of remapping observed in individuals. Remapping pairs were identified based on the sameness of the waveform recorded during several sessions (see Figs. 1, 2 and Materials and Methods). $\boldsymbol{b}$, Frequency distributions of average place field correlations within (stable rats, $\mathbf{0}$; remapping rats, (2) and between (stable rats, (3; remapping rats, (4) freely moving sessions in the square arena. Insets show the same distributions for the non-delayed units (blue) and for the delayed units (green; axes are downscaled 2-fold). For both stable and remapping animals, the distributions of within correlations were unimodal and equivalent (Mann-Whitney $U$ test, $Z=-0.575$, NS), reflecting a high degree of consistency of the place fields within sessions. The distribution of between correlations is unimodal for stable rats; the remapping rats' distribution is bimodal $(Z=15.870, p<0.001)$, primarily correlated with the delayed activity in at least one unit of the pair considered (for details, see Fig. 6 and Results). c, Distribution of individual variance scores in stable and remapping rats (1; close-up in 2). Note the presence toward lowest values of the remapping rats' distributions. $\boldsymbol{d}$, There were no differences in the standardized firing rates distributions of the middle-aged (left) and the aged (middle) rats, as can be seen with the completely overlapping cumulative probability functions of the two distributions (right). The gray area is a close-up of the two curves between the standardized firing rate values of 2 and 10 for a probability comprised between 0.9 and 1.e, As suggested previously in c, the standardized firing rate distributions of the stable (left) and remapping (middle) rats are significantly different as highlighted by the divergence of the two cumulative probability distributions (right). The close-up shows a clear dissociation of the two cumulative probability functions $\left({ }^{* * *} p<0.001\right)$; the one extracted from the remapping rats' data is steeper as the variance is lower (for a similar phenomenon, see Fenton et al., 2010). Removing the two outliers (previously identified in $c$ ) from the stable rats' data $\left(\sigma^{2}=\right.$ $\left.5.073, \mu=0.03, N_{\text {passes }}=8531\right)$ still leaves a significant difference between the two distributions $(W=22.359, p<0.001)$. See also Table 1.

performance curve shows a decrease in mean percentage correct performance as a function of delay. Figure $8 b$ shows the correlation between interindividual variability expressed as SEM and the length of the delay. This variability increases in a linear manner with the duration of the delay $\left(r^{2}=0.87, p<0.01\right)$ as highlighted by the spread of individual behavioral scores (Fig. 8c) for long delays (second column) when compared with short delays (first column). Individual performance curves for six rats are shown in Figure $8 d$; the remapping status of the two remaining rats that were used in this analysis could not be assessed because of the lack of pairs isolated (for details, see Table 1). Interestingly, the two remapping rats (black triangles in the top right corner of each 
graph) display a clear impairment at the longest delays (26-30 s), with the percentage of correct responses at chance (rat rd, binomial $Z$ ratio $=-0.16$, NS; rat rh, binomial $Z$ ratio $=0$, NS). Correlating the overdispersion values with overall performance for the eight rats yields a nonsignificant positive trend $\left(r^{2}=0.40\right.$, NS; Fig. $8 e$, left). The same correlation for short delays $(1-5 \mathrm{~s})$ is not statistically significant $\left(r^{2}=\right.$ 0.04 , NS; Fig. $8 e$, middle). This contrasts strongly with the significant positive correlation $\left(r^{2}=0.65, p<0.05\right.$; Fig. $8 e$, right) between overdispersion and the long delays (26-30 s) that are hippocampus dependent (Hampson et al., 1999) and sensitive to aging (Dunnett et al., 1988). Together, these results show that a strong linear relationship exists between place cell firing variability and behavioral performance, suggesting that overdispersion is an accurate predictor of an individual's cognitive capacities in a hippocampus-dependent memory task.

\section{Discussion}

\section{Late-onset activity is correlated with the global remapping} process in old rats

Late-onset activity occurring during exploration of a new environment was first noted by Hill (1978), in which 2 place cells of 12 recorded exhibited a delay in their firing during the rat's initial exposure to the maze. Since then, the phenomenon has been little explored: Tanila et al. (1997b) reported one example of a lateonset place cell recorded in a young rat, and Leutgeb et al. (2004) similarly described diachronic differences in information content during the global remapping process. Firing onset delays were also reported in another study by Tanila et al. (1997a) when taking into account age category and cognitive status, the firing onset being defined in this case as the "number of trials completed before firing appears." This variable was on average one or two laps for old memory-impaired rats and two or three laps for old memory-intact rats and young rats. Given its rarity in other studies (Mehta et al., 1997; Shen et al., 1997), it has been suggested (Barnes, 1998) that these firing onset differences could be linked to the nature of reward (i.e., medial forebrain bundle stimulation) used in the experiment of Tanila et al. We show here that late firing onset does occur in old rats in physiological conditions and that time as the sole variable can deceptively characterize this feature. Although no indication has been given whether the groups were different in their locomotion, it is likely that the old memory-impaired rats identified in the study by Tanila et al. showed increased exploration during the first phase of the session (resulting therefore in a shorter onset when compared with the old memory-intact and young animals). We also demonstrated that delayed activity is present specifically when hippocampal representations are unstable from one session to the other. This strong positive correlation between the proportion of delayed units and the proportion of remapping observed in individuals is attributable to the fact that late-onset activity is primarily observed during remapping episodes and not when units are stable from one session to the other. This late place field development has a crucial impact on how place cell activity is assessed in old rats. As stated in the Introduction, the basic properties of place cells are not affected by aging (Barnes et al., 1983; Markus et al., 1994; Mizumori et al., 1996; Mizumori and Kalyani, 1997). The a
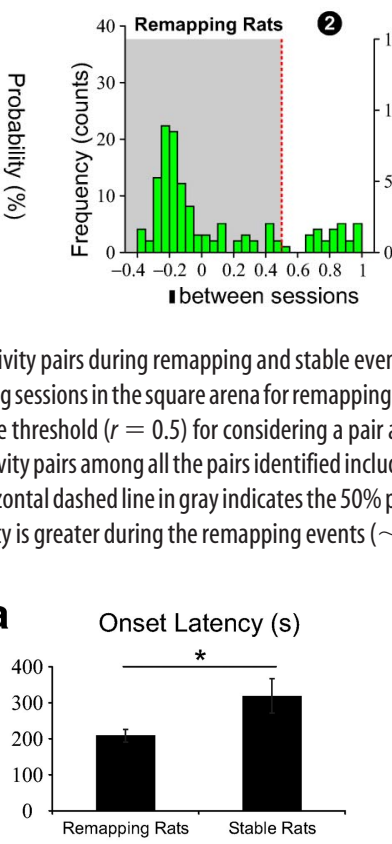

b

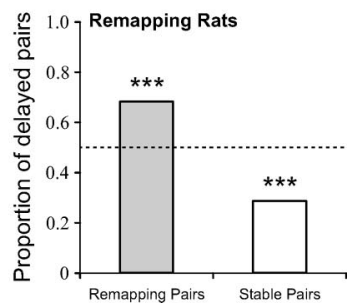

b

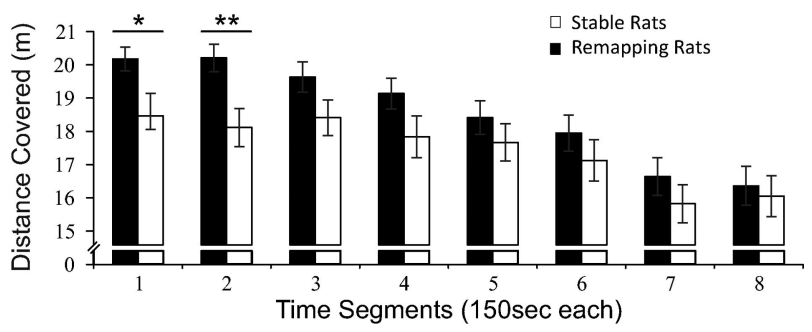

Figure 7. Additional characterization of late-onset firing in individuals. $\boldsymbol{a}$, Plots of the onset latency and onset pass number showing conflicting views on putative differences in firing onset between remapping and stable rats. Although delayed units recorded from the remapping rats tend to fire before the delayed population from the stable animals ( ${ }^{*} p<0.05$ ), no difference is seen between the two groups when comparing the number of passes elapsed before firing onset. $\boldsymbol{b}$, Differences in locomotion between remapping and stable rats occurring at the beginning of the freely moving session. The distance covered by the remapping rats during the two first 150-s-long segments is significantly increased when compared with the one covered by the stable animals ( ${ }^{*} p<0.05 ;{ }^{* *} p<0.01$ ). This last result might explain why the latency is significantly reduced in the remapping group, whereas the number of passes before firing onset remains unchanged. Note that the decreased exploration in remapping rats during the third time segment coincide with the average latency before onset firing, suggesting therefore that place cell activation at that time may mark the end of the remapping process per se.

notable exception is average activity, which was decreased in old rats in one study (Shen et al., 1997). The spatial specificity of place cells in old animals was either decreased or increased in some studies (Barnes et al., 1983; Mizumori et al., 1996; Shen et al., 1997; Tanila et al., 1997a,b) but not affected in others (Markus et al., 1994; Barnes et al., 1997) when compared with young animals. These discrepancies have been ascribed to differences in task demands and to the nature of the hippocampal subregions being analyzed (Barnes, 1998). Conversely, the absence of firing for several minutes that we isolated in our study alters numerous spatial indices commonly used for characterizing place cell signal integrity (e.g., average firing activity, infield firing rate, and spatial information content). We suggest therefore that this particular activity should be identified and isolated in future studies to provide an accurate account for aged-related place cell impairments. 

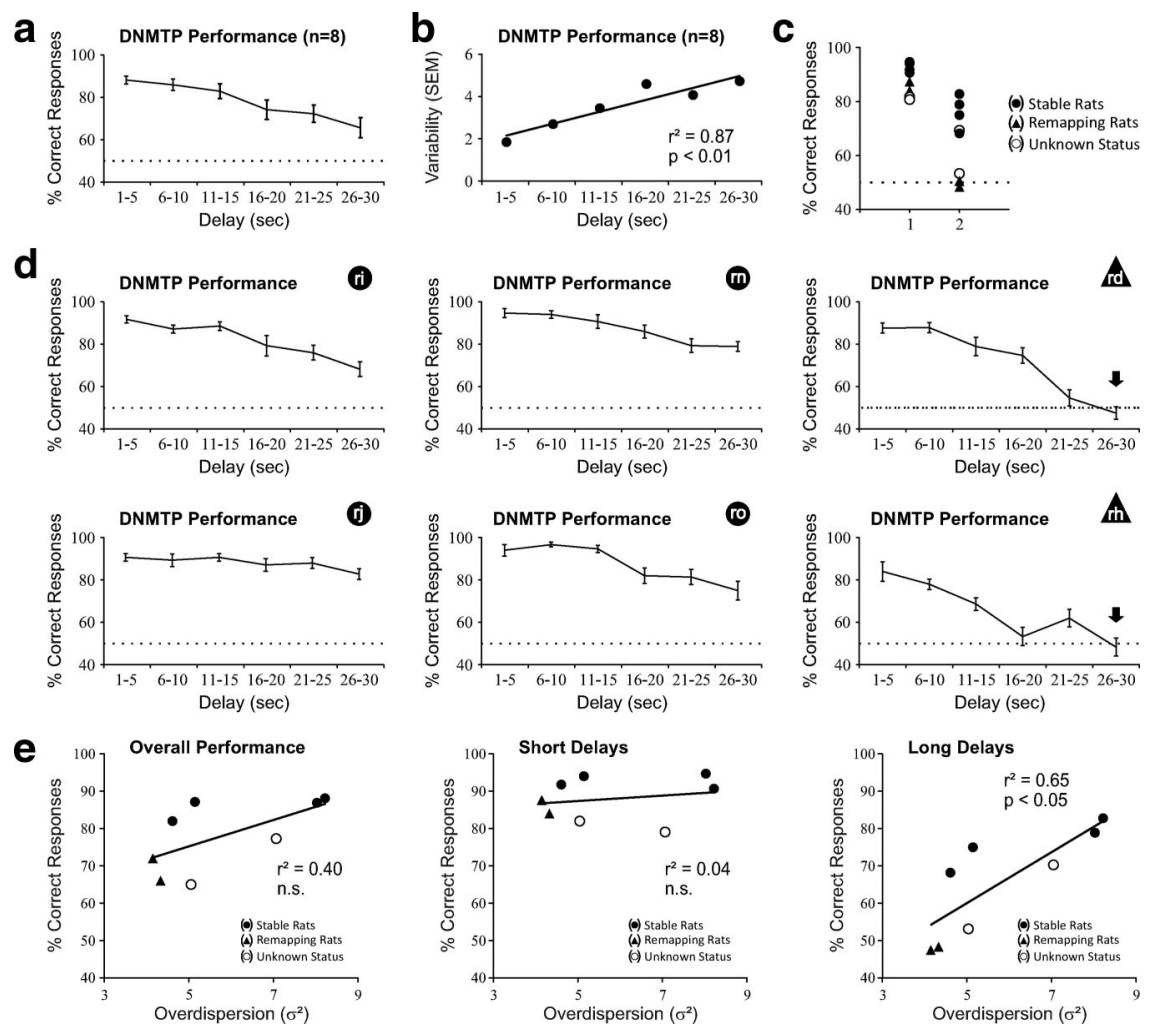

Figure 8. Individually assessed memory performance is correlated with the degree of overdispersion. $\boldsymbol{a}$, The curve shows the DNMTP performance calculated over 10 sessions of 90 trials each, presenting the typical decrease in mean \pm SEM percentage correct performance as a function of delay (1-30 s interval in 5 s increments) for eight rats. The dashed line indicates chance level of performance. $\boldsymbol{b}$, Correlation between interindividual variability as expressed by SEM and the duration of the delay. This graph shows a strong positive relationship between behavioral variability and the length of the delay $\left(r^{2}=0.87, p<0.01\right)$. $c$, As suggested in $\boldsymbol{b}$, individual behavioral scores show more spread at the longest delays ( $26-30 \mathrm{~s}$; second column) when compared with short delays ( $1-5 \mathrm{~s}$; first column). $\boldsymbol{d}$, Individual performance curves for six rats ( 4 non-remapping rats and 2 remapping rats; individual identification codes appear in the black circles and triangles). Note that the two remapping rats (black triangles) show a dramatic decrease of performance toward the longest delays (black arrows) not different from chance level. $\boldsymbol{e}$, Correlations between performance and overdispersion per individual. A trend for positive correlation between the degree of overdispersion and the overall performance can be seen on the left $\left(r^{2}=0.40, N S\right)$. Considering the shortest delays $(1-5 \mathrm{~s}$; which are typically resistant to hippocampal disruption) reduces drastically the strength of the correlation $\left(r^{2}=0.04\right.$, NS), whereas taking into account the long delays (26-30s; which are typically sensitive to hippocampal disruption) reveals a strong positive correlation $\left(r^{2}=0.65, p<\right.$ $0.05)$. This last result indicates that overdispersion is a good predictor of individual cognitive state and hence performance on the DNMTP task.

\section{Overdispersion and synaptic plasticity}

As pointed out by Burke and Barnes $(2006,2010)$, there is no loss of synaptic contacts in CAl during aging, contrary to what is found in the dentate gyrus and CA3 (Smith et al., 2000). This is true for both the layer III entorhinal cortex-CA1 synapse (Smith et al., 2000) and the Schaffer collateral-CA1 synapse (Geinisman et al., 2004). However, it has been demonstrated that the postsynaptic density (PSD) areas of the Schaffer collateral perforated axospinous synapses is significantly decreased in learning-impaired aged rats when compared with young animals (Nicholson et al., 2004). These perforated synapses are thought to play an important role in synaptic plasticity thanks to their multiple partitioned transmission zones (Geinisman, 1993; Nicholson and Geinisman, 2009). In an early attempt to model the mechanisms underlying CA1 output overdispersion, Olypher et al. (2002) suggested that this variability could be ascribed to random inhibition affecting a subset of synaptic inputs. In this context, the synaptic loss of efficacy attributable to the decrease of the PSD in CA1 might lead to the reduction of time variability in the inputs, resulting in a reduction of the overdispersion.
Bimodal standardized firing rate variations distribution as a functional signature for global remapping Standardized firing rate distributions are classically unimodal, with a peak at approximately zero, as first described by Fenton and Muller (1998; see also Olypher et al., 2002). However, Jackson and Redish (2007) found clear bimodal distributions with a sharp peak at negative standardized firing rates on multiple occasions in different behavioral contexts. In this latter report, food-deprived rats were trained in several tasks, including shuttling back and forth along a linear track, foraging for scattered pellets in a circular arena, and navigating to an invisible goal for food reward in the same arena. Importantly, recording sessions took place in the same room and consisted of $20 \mathrm{~min}$ exposure to each of the three tasks chosen each day in a random manner. The authors explained this peak at negative values by the presence of a large number of silent passes (no firing) while recording the place cell activity. Based on their map-switching hypothesis to explain overdispersion, they attributed these nofire passes to strong rate differences between internal representations. Conversely, Markus et al. (1995) showed that different place cell responses within the same environment could be induced by fluctuations in the task reward contingencies within session. In our study, however, the animals were trained in one room in one single task always using the same arena, excluding any task reward contingency ambiguity as a possible source of conflict in the internal states. We argue therefore that the bimodal standardized firing rates distribution observed in the study by Jackson and Redish and in the present work is linked to the late-onset firing of a fraction of place cells that co-occur with the global remapping process.

\section{Overdispersion degree predicts individual cognitive performance}

Two previous studies found a clear (negative) correlation between overdispersion observed in CA1 place cells and the cognitive load of the behavioral task in which the animals are engaged (Jackson and Redish, 2007; Fenton et al., 2010). Both studies argue that place cell variability reflects changes in active cell assemblies, whether these changes are attributable to attentional reference-frame shift (Olypher et al., 2002; Fenton et al., 2010; Kelemen and Fenton, 2010) or to a more general internal mapswitching process (Jackson and Redish, 2007). We, however, found a positive correlation between place cell firing variability and the cognitive load of the behavioral task, as assessed by the behavioral performance toward long delays in the DNMTP task. These apparent contradictory results are not mutually exclusive because animals trained in the goal-directed task, which was used 
in the studies of both Jackson and Redish and Fenton et al., might have individually shown exactly the same correlation between performance level and place cell variability. The correlation between place cell variability and performance level mirrors in some way previous results obtained by Barnes (1979) showing that an individual rat's performance in a circular platform task was correlated with the persistence of enhancement of the granule cells synaptic responses. This last observation strengthens the idea that overdispersion modifications observed in our study might be directly linked to a loss of synaptic excitability, thus reflecting a structural modification of the hippocampal network rather than a functional consequence of the remapping process per se. This constitutive alteration of the network would then explain the strong relationship we observed between overdispersion and behavioral performance despite the orthogonalization of representations of the two behavioral tasks. Additional work will be required, however, to fully elucidate the consequences of unstable hippocampal representations on behavioral performance in hippocampus-dependent tasks. Recent studies suggest that trial-specific information in the DNMTP task is encoded accurately by hippocampal spatiotemporal firing patterns (Deadwyler and Hampson, 2004, 2006). In a follow-up study, Berger et al. (2011) showed that cognitive enhancement could be achieved through neuronal stimulation based on such spatiotemporal firing patterns during the sample response. Together, these results suggest that the fine temporal dynamics recorded in CA1 during DNMTP tasks and the map-switching process observed in various spatial navigation tasks (Jackson and Redish, 2007; Fenton et al., 2010) subserve the same function. In this view, a high degree of firing variability might reflect the necessary spatiotemporal organization of hippocampal neuronal activity to successfully encode and maintain useful information over long delays.

\section{References}

Barnes CA (1979) Memory deficits associated with senescence: a neurophysiological and behavioral study in the rat. J Comp Physiol Psychol 93:74-104.

Barnes CA (1998) Spatial cognition and functional alterations of aged rat hippocampus. In: Handbook of the aging brain (Wang E, Snyder DS, eds), pp 51-66. New York: Academic.

Barnes CA, McNaughton BL, O’Keefe J (1983) Loss of place specificity in hippocampal complex spike cells of senescent rat. Neurobiol Aging 4:113-119.

Barnes CA, Suster MS, Shen J, McNaughton BL (1997) Multistability of cognitive maps in the hippocampus of old rats. Nature 388:272-275.

Berger TW, Hampson RE, Song D, Goonawardena A, Marmarelis VZ, Deadwyler SA (2011) A cortical neural prosthesis for restoring and enhancing memory. J Neural Eng 8:046017.

Burgess N, Cacucci F, Lever C, O’Keefe J (2005) Characterizing multiple independent behavioral correlates of cell firing in freely moving animals. Hippocampus 15:149-153.

Burke SN, Barnes CA (2006) Neural plasticity in the ageing brain. Nat Rev Neurosci 7:30-40.

Burke SN, Barnes CA (2010) Senescent synapses and hippocampal circuit dynamics. Trends Neurosci 33:153-161.

Cacucci F, Lever C, Wills TJ, Burgess N, O'Keefe J (2004) Theta-modulated place-by-direction cells in the hippocampal formation in the rat. J Neurosci 24:8265-8277.

Deadwyler SA, Hampson RE (2004) Differential but complementary mnemonic functions of the hippocampus and subiculum. Neuron 42:465-476.

Deadwyler SA, Hampson RE (2006) Temporal coupling between subicular and hippocampal neurons underlies retention of trial-specific events. Behav Brain Res 174:272-280.

Dunnett SB, Evenden JL, Iversen SD (1988) Delay-dependent short-term memory deficits in aged rats. Psychopharmacology (Berl) 96:174-180.

Fenton AA, Muller RU (1998) Place cell discharge is extremely variable dur- ing individual passes of the rat through the firing field. Proc Natl Acad Sci USA 95:3182-3187.

Fenton AA, Csizmadia G, Muller RU (2000) Conjoint control of hippocampal place cell firing by two visual stimuli. I. The effects of moving the stimuli on firing field positions. J Gen Physiol 116:191-209.

Fenton AA, Kao HY, Neymotin SA, Olypher A, Vayntrub Y, Lytton WW, Ludvig N (2008) Unmasking the CA1 ensemble place code by exposures to small and large environments: more place cells and multiple, irregularly arranged, and expanded place fields in the larger space. J Neurosci 28:11250-11262.

Fenton AA, Lytton WW, Barry JM, Lenck-Santini PP, Zinyuk LE, Kubík S, Bures J, Poucet B, Muller RU, Olypher AV (2010) Attention-like modulation of hippocampus place cell discharge. J Neurosci 30:4613-4625.

Gallagher M, Burwell R, Burchinal M (1993) Severity of spatial learning impairment in aging: development of a learning index for performance in the Morris water maze. Behav Neurosci 107:618-626.

Geinisman Y (1993) Perforated axospinous synapses with multiple, completely partitioned transmission zones: probable structural intermediates in synaptic plasticity. Hippocampus 3:417-433.

Geinisman Y, Ganeshina O, Yoshida R, Berry RW, Disterhoft JF, Gallagher M (2004) Aging, spatial learning, and total synapse number in the rat CA1 stratum radiatum. Neurobiol Aging 25:407-416.

Grossman SE, Fontanini A, Wieskopf JS, Katz DB (2008) Learning-related plasticity of temporal coding in simultaneously recorded amygdalacortical ensembles. J Neurosci 28:2864-2873.

Hampson RE, Jarrard LE, Deadwyler SA (1999) Effects of ibotenate hippocampal and extrahippocampal destruction on delayed-match and -nonmatch-to-sample behavior in rats. J Neurosci 19:1492-1507.

Hill AJ (1978) First occurrence of hippocampal spatial firing in a new environment. Exp Neurol 62:282-297.

Ingram DK (1988) Complex maze learning in rodents as a model of agerelated memory impairment. Neurobiol Aging 9:475-485.

Jackson J, Redish AD (2007) Network dynamics of hippocampal cellassemblies resemble multiple spatial maps within single tasks. Hippocampus 17:1209-1229.

Johnson A, Fenton AA, Kentros C, Redish AD (2009) Looking for cognition in the structure within the noise. Trends Cogn Sci 13:55-64.

Kelemen E, Fenton AA (2010) Dynamic grouping of hippocampal neural activity during cognitive control of two spatial frames. PLoS Biol 8:e1000403.

Leutgeb JK, Leutgeb S, Moser MB, Moser EI (2007) Pattern separation in the dentate gyrus and CA3 of the hippocampus. Science 315:961-966.

Leutgeb S, Leutgeb JK, Treves A, Moser MB, Moser EI (2004) Distinct ensemble codes in hippocampal areas CA3 and CA1. Science 305:1295-1298.

Markus EJ, Barnes CA, McNaughton BL, Gladden VL, Skaggs WE (1994) Spatial information content and reliability of hippocampal CA1 neurons: effects of visual input. Hippocampus 4:410-421.

Markus EJ, Qin YL, Leonard B, Skaggs WE, McNaughton BL, Barnes CA (1995) Interactions between location and task affect the spatial and directional firing of hippocampal neurons. J Neurosci 15:7079-7094.

McNaughton BL, Barnes CA, O'Keefe J (1983) The contributions of position, direction, and velocity to single unit activity in the hippocampus of freely-moving rats. Exp Brain Res 52:41-49.

Mehta MR, Barnes CA, McNaughton BL (1997) Experience-dependent, asymmetric expansion of hippocampal place fields. Proc Natl Acad Sci USA 94:8918-8921.

Mizumori SJ, Kalyani A (1997) Age and experience-dependent representational reorganization during spatial learning. Neurobiol Aging 18:651-659.

Mizumori SJ, Lavoie AM, Kalyani A (1996) Redistribution of spatial representation in the hippocampus of aged rats performing a spatial memory task. Behav Neurosci 110:1006-1016.

Muller RU, Kubie JL (1987) The effects of changes in the environment on the spatial firing of hippocampal complex-spike cells. J Neurosci 7:1951-1968.

Muller RU, Kubie JL (1989) The firing of hippocampal place cells predicts the future position of freely moving rats. J Neurosci 9:4101-4110.

Muller RU, Kubie JL, Ranck JB Jr (1987) Spatial firing patterns of hippocampal complex-spike cells in a fixed environment. J Neurosci 7:1935-1950. 
Muller RU, Bostock E, Taube JS, Kubie JL (1994) On the directional firing properties of hippocampal place cells. J Neurosci 14:7235-7251.

Nicholson DA, Geinisman Y (2009) Axospinous synaptic subtype-specific differences in structure, size, ionotropic receptor expression, and connectivity in apical dendritic regions of rat hippocampal CA1 pyramidal neurons. J Comp Neurol 512:399-418.

Nicholson DA, Yoshida R, Berry RW, Gallagher M, Geinisman Y (2004) Reduction in size of perforated postsynaptic densities in hippocampal axospinous synapses and age-related spatial learning impairments. J Neurosci 24:7648-7653.

O'Keefe J, Conway DH (1978) Hippocampal place units in the freely moving rat: why they fire where they fire. Exp Brain Res 31:573-590.

O'Keefe J, Dostrovsky J (1971) The hippocampus as a spatial map. Preliminary evidence from unit activity in the freely-moving rat. Brain Res 34:171-175.

Olypher AV, Lánský P, Fenton AA (2002) Properties of the extra-positional signal in hippocampal place cell discharge derived from the overdispersion in location-specific firing. Neuroscience 111:553-566.

Rapp PR, Amaral DG (1992) Individual differences in the cognitive and neurobiological consequences of normal aging. Trends Neurosci 15:340-345.

Shen J, Barnes CA, McNaughton BL, Skaggs WE, Weaver KL (1997) The effect of aging on experience-dependent plasticity of hippocampal place cells. J Neurosci 17:6769-6782.

Skaggs WE, McNaughton BL, Gothard KM (1993) An information- theoretic approach to deciphering the hippocampal code. In: Advances in neural information processing systems (Hanson SJ, Cowan JD, Giles CL, eds), pp 1030-1037. San Mateo, CA: Morgan Kaufmann.

Smith TD, Adams MM, Gallagher M, Morrison JH, Rapp PR (2000) Circuit-specific alterations in hippocampal synaptophysin immunoreactivity predict spatial learning impairment in aged rats. J Neurosci 20:6587-6593.

Tanila H, Shapiro M, Gallagher M, Eichenbaum H (1997a) Brain aging: changes in the nature of information coding by the hippocampus. J Neurosci 17:5155-5166.

Tanila H, Sipilä P, Shapiro M, Eichenbaum H (1997b) Brain aging: impaired coding of novel environmental cues. J Neurosci 17:5167-5174.

Thompson LT, Best PJ (1990) Long-term stability of the place-field activity of single units recorded from the dorsal hippocampus of freely behaving rats. Brain Res 509:299-308.

Wells CE, Krikke B, Saunders J, Whittington A, Lever C (2009) Changes to open field surfaces typically used to elicit hippocampal remapping elicit graded exploratory responses. Behav Brain Res 197:234-238.

Wilson IA, Ikonen S, McMahan RW, Gallagher M, Eichenbaum H, Tanila H (2003) Place cell rigidity correlates with impaired spatial learning in aged rats. Neurobiol Aging 24:297-305.

Wilson MA, McNaughton BL (1993) Dynamics of the hippocampal ensemble code for space. Science 261:1055-1058. 